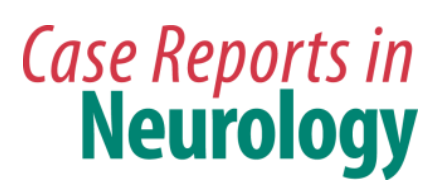

\title{
Resolution of Cystic Enhancement to Add-On Tumor Treating Electric Fields for Recurrent Glioblastoma after Incomplete Response to Bevacizumab
}

\author{
Grace Elzinga Eric T. Wong \\ Brain Tumor Center and Neuro-Oncology Unit, Beth Israel Deaconess Medical Center, \\ Boston, Mass., USA
}

\section{Key Words}

Glioblastoma $\cdot$ Alternating electric fields $\cdot$ Response

\begin{abstract}
The NovoTTF-100A device emits alternating tumor treating electric fields (TTFields) that interfere with cytokinesis and chromosome segregation during mitosis. Because it has a similar efficacy to cytotoxic chemotherapy, the device has been approved by the United States Food and Drug Administration for the treatment of recurrent glioblastoma. Although bevacizumab has been in use for recurrent glioblastoma, patients who experience incomplete or no response to bevacizumab may be predisposed to early bevacizumab treatment failure. However, the addition of TTFields therapy may augment the efficacy from bevacizumab. We report a patient with recurrent cystic glioblastoma who received add-on TTFields therapy due to an incomplete response to single-agent bevacizumab. After 6 cycles of therapy, a resolution of cystic enhancement was noted, together with reduction of the tumor cyst and resolution of most of the cerebral edema in the surrounding brain. However, the patient also suffered from relapsed disease at locations distant from the original glioblastoma and the corresponding radiation fields received at initial diagnosis. We conclude that combination TTFields and bevacizumab therapy is safe and may be efficacious for patients with recurrent glioblastoma. A further study would be needed to determine the relapse pattern and the distribution of the electric fields in the brain.

(c) 2014 S. Karger AG, Basel
\end{abstract}




\section{Introduction}

Recurrent glioblastoma has a poor prognosis. Patients treated with salvage chemotherapy had a response rate of only $9 \%$ and a median time to tumor progression (TTP) of 9 weeks [1]. Although bevacizumab can offer a significantly higher response rate of $55 \%$ and a period of clinical stabilization with a median TTP of 26 weeks, the tumor remains in the brain and continues to proliferate despite clinical and radiological appearances of improvement [2]. As a result, bevacizumab has a questionable impact on the overall survival of patients $[3,4]$. Therefore, new and novel treatments are needed for patients with recurrent glioblastoma who failed initial treatment with radiotherapy and temozolomide.

The NovoTTF-100A device is a new treatment approved by the United States Food and Drug Administration (FDA) for recurrent glioblastoma. The device emits alternating tumor treating electric fields (TTFields) via 2 pairs of transducer arrays placed orthogonally on the scalp. The TTFields work by interrupting tumor cells during mitosis, resulting in violent blebbing during cytokinesis, asymmetric chromosome segregation, and aneuploidy $[5,6]$. These cell biology effects ultimately result in apoptosis or slippage into a G0 state of the tumor cell, while simultaneously making it susceptible to immunogenic cell death [6]. In the pivotal phase III clinical trial, the NovoTTF-100A device had a similar efficacy when compared to salvage chemotherapy, but without the toxicities associated with systemic chemotherapies [7, 8]. Here, we report a patient who had failed bevacizumab therapy for a recurrent cystic glioblastoma and, at the time of bevacizumab continuation, received add-on TTFields therapy by using the NovoTTF-100A device. This treatment combination eventually resulted in the disappearance of cystic enhancement together with a marked reduction of the cyst size and the cerebral edema in the surrounding brain.

\section{Case Report}

The patient is a 76-year-old right-handed woman who came to the Brain Tumor Center for an evaluation of her recurrent glioblastoma after bevacizumab failure. Her initial neurological problems occurred 9 months prior to presentation and consisted of mental confusion and comprehension problems manifesting as a fluent aphasia. A gadoliniumenhanced head MRI at an outside hospital showed a cystic enhancing mass in the left temporal lobe of the brain. She underwent a neurosurgical resection, and the pathology demonstrated glioblastoma. She then received 6 weeks of external beam fractionated radiotherapy to 6,000 cGy (200 cGy in 30 fractions) with concomitant daily temozolomide at $75 \mathrm{mg} / \mathrm{m}^{2}$, followed by adjuvant temozolomide $200 \mathrm{mg} / \mathrm{m}^{2}$ for 5 days on a monthly basis. After 5 cycles of adjuvant temozolomide, new cystic enhancement was discovered while performing a head MRI (fig. 1a, b), and she was placed on $4 \mathrm{mg}$ of dexamethasone 4 times/day. Bevacizumab was subsequently started at a dose of $10 \mathrm{mg} / \mathrm{kg}$ every 2 weeks. After 2 cycles of bevacizumab, there was only a partial decrease in the gadolinium enhancement and the size of the cystic tumor (fig. 1c, d). She then came to our institution and her original histological diagnosis was confirmed. Additional pathology testing revealed a negative IDH1 immunohistochemical labeling but a positive OLIG2, EGFR amplification and methylated MGMT promotor status. Because TTFields interrupt tumor cells during mitosis and have no appreciable overlapping toxicity with bevacizumab, we proceeded to add to her bevacizumab treatment TTFields therapy using the NovoTTF-100A device. Each cycle consists of 4 weeks of continuous treatment. The treatment compliance was recorded by sensors, embedded within the transducer arrays and can be downloaded by computer for 
review in clinic visits. Her dexamethasone was weaned off after 2 cycles of combined treatment. After a total of 6 cycles of bevacizumab plus TTFields therapy, with a respective mean and median compliance of 17.6 hours (73\% of the day) and 18.4 hours ( $77 \%$ of the day) (range 3.6 to $22.8 \mathrm{~h}$ ), there was a near complete resolution of gadolinium enhancement and a $65 \%$ reduction in the size of the cystic tumor (fig. 1e, f). However, there were also recurrent tumors detected in the left internal capsule and the medial left frontal brain (fig. $2 \mathrm{a}, \mathrm{b})$, which were located outside of the prior radiation treatment fields. Therefore, the recurrent tumors were treated with fractionated CyberKnife radiosurgery to 2,100 cGy (700 cGy in 3 fractions). Despite this radiosurgery intervention, the patient continued to deteriorate neurologically and her deterioration was most likely caused by the microscopic invasive glioblastoma. She eventually died 15 months after her first recurrence and 22 months after initial diagnosis. Her health care proxy did not grant an autopsy.

\section{Discussion}

The addition of NovoTTF-100A to bevacizumab therapy in our patient appears to be safe and may provide added efficacy after initial incomplete response to bevacizumab alone. The rationales to combine TTFields therapy with bevacizumab are threefold. First, there is no overlapping side effect and, therefore, this combination does not appear to cause synergistic or additive toxicities. In patients with glioblastoma, the most dreaded complication from bevacizumab is hemorrhage within the tumor. We retrospectively analyzed 20 patients treated with this combination and found no instance of intracranial hemorrhage [9]. Other treatment side effects are minor in severity and they include the expected scalp rash, electric shock sensation from poorly applied transducer arrays and vivid dreams, all of which resolved upon the application of corticosteroid cream and adjustment of the arrays. Second, TTFields therapy mimics the effects of chemotherapy by interference with tumor cell mitosis while not having the conventional side effects of chemotherapy [5, 7]. When first approved by the FDA, bevacizumab was combined with various cytotoxic agents, including carboplatin and irinotecan [10], carboplatin and etoposide [11] as well as lomustine or carmustine [12], but the side effects do not warrant the routine clinical use of bevacizumab combined with cytotoxic chemotherapy. Third, both bevacizumab and NovoTTF-100A are listed in the National Comprehensive Cancer Network practice guideline for recurrent glioblastoma. Therefore, there appear to be strong rationales to combine NovoTTF-100A with bevacizumab for recurrent glioblastoma.

The resolution of our patient's cystic tumor is a notable response to NovoTTF-100A and bevacizumab after initial bevacizumab failure. Although the response assessment in neurooncology criteria deem it as nonmeasurable, cystic tumors cannot be measured in a traditional bidimensional fashion for response assessment, unless there is an associated solid nodule measuring $10 \mathrm{~mm}$ or greater in diameter [13]. However, the disappearance of enhancement in the cystic tumor is still remarkable. In their retrospective series of 51 recurrent high-grade gliomas treated with bevacizumab and irinotecan, Zuniga et al. [14] reported only $8 \%$ of patients with a complete response while the majority had either a partial response (63\%) or no response (29\%). Similarly, when bevacizumab was used as a single agent, Kreisl et al. [15] and Friedman et al. [16] reported an even less robust complete response rate of 2 and $1 \%$, respectively. These data indicate that bevacizumab alone rarely results in a complete radiographic response, and the partial response seen in a majority of patients suggests that there are probably multiple proangiogenic pathways activated in the 
glioblastoma. Therefore, the addition of TTFields therapy may augment the efficacy of bevacizumab.

The efficacy of combination TTFields and bevacizumab therapy is unknown. A prior post hoc analysis of the response characteristics from the pivotal phase III trial indicates that secondary glioblastomas as well as low or no dexamethasone usage are potentially important predictors of response in patients treated with the NovoTTF-100A device alone $[7,17]$. First, secondary glioblastomas may have a different genetic composition that makes the tumor cells more susceptible to TTFields. Indeed, Verhaak et al. [18] have shown that most of the secondary glioblastomas fall in the proneural genotype with amplification of PDGFRA and OLIG2 as well as mutations in IDH1 and TP53. Although our patient's tumor appears to be a primary glioblastoma by the lack of IDH1 immunohistochemical labeling, there could still be unidentified genetic mutations that make her tumor susceptible to TTFields therapy. Indeed, 9 out of 14 responders in the phase III trial did not have prior lowgrade histologies, and they could have genetic mutations or epigenetic alterations that make them susceptible to NovoTTF-100A monotherapy [17]. Second, the slower growth rate of our patient's tumor may have helped to allow sufficient time for the TTFields to effect a treatment response. This is because the median time to response for TTFields therapy is longer than that for chemotherapies, 8.4 versus 5.8 months, respectively, as noted in the prior post hoc response analysis [17]. Last, our patient's dexamethasone was completely discontinued 2 months after initiation of combination TTFields and bevacizumab therapy, but a near complete resolution of gadolinium enhancement and a significant reduction of the cystic tumor were not detectable until 6 months into treatment. Consistent with this observation, the inverse relationship between response and dexamethasone dosage is probably a consequence of removing the immunosuppressive effect of dexamethasone, which would allow for better control of the glioblastoma by the patient's immune system. Therefore, allowing sufficient treatment time and removing dexamethasone are key parameters to maximize the probability of a response from TTFields.

The pattern of relapse from combination TTFields and bevacizumab therapy is unknown. At the time of tumor recurrence, our patient's new disease was located outside of her prior radiation field. This type of distant recurrence of glioblastoma could be the consequence of progressive development of the invasive phenotype, intracranial inhomogeneity of the TTFields, or incomplete coverage of certain parts of the brain by TTFields. These hypothesis-generating observations would be important for future studies to correlate the location of the relapsed disease and the distribution of the electric fields within the brain.

\section{References}

1 Wong ET, Hess KR, Gleason MJ, Jaeckle KA, Kyritsis AP, Prados MD, et al: Outcomes and prognostic factors in recurrent glioma patients enrolled onto phase II clinical trials. J Clin Oncol 1999;17:2572-2578.

-2 Wong ET, Gautam S, Malchow C, Lun M, Pan E, Brem S: Bevacizumab for recurrent glioblastoma multiforme: a meta-analysis. J Natl Compr Canc Netw 2011;9:403-407.

-3 Reardon DA, Herndon JE, Peters KB, Desjardins A, Coan A, Lou E, et al: Bevacizumab continuation beyond initial bevacizumab progression among recurrent glioblastoma patients. Br J Cancer 2012;107:1481-1487.

4 Iwamoto FM, Fine HA: Bevacizumab for malignant gliomas. Arch Neurol 2010;67:285-288.

5 Lee SX, Wong ET, Swanson KD: Mitosis interference of cancer cells by NovoTTF-100A causes decreased cellular viability (abstract); in Proceedings of the 104th Annual Meeting of the American Association for Cancer Research, 6-10 April, 2013, Washington, DC. Cancer Res 2013;73(8suppl):abstract No. 709. DOI:10.1158/1538-7445.AM2013-709.

6 Lee DX, Wong E, Swanson K: Disruption of cell division within anaphase by tumor treating electric fields (TTFields) leads to immunogenic cell death. Neuro Oncol 2013;15(suppl 3):iii66-iii67. 


\begin{tabular}{l|l}
\hline Case Rep Neurol 2014;6:109-115 & \\
\hline DOI: $10.1159 / 000362264$ & $\begin{array}{l}\text { ○ 2014 S. Karger AG, Basel } \\
\text { www.karger.com/crn }\end{array}$ \\
\hline
\end{tabular}

Elzinga et al.: Resolution of Cystic Enhancement to Add-On Tumor Treating Electric Fields for Recurrent Glioblastoma after Incomplete Response to Bevacizumab

7 Stupp R, Wong ET, Kanner AA, Steinberg D, Engelhard H, Heidecke V, et al: NovoTTF-100A versus physician's choice chemotherapy in recurrent glioblastoma: A randomized phase III trial of a novel treatment modality. Eur J Cancer 2012;48:2192-2202.

-8 Fonkem E, Wong ET: NovoTTF-100A: a new treatment modality for recurrent glioblastoma. Expert Rev Neurother 2012;12:895-899.

9 Elzinga G, Chung AT, Wong E: Safety analysis of bevacizumab plus NovoTTF-100A in patients with recurrent malignant gliomas (abstract 2082). J Clin Oncol 2013;31(suppl 15):abstract No. 2082.

-10 Reardon DA, Desjardins A, Peters KB, Gururangan S, Sampson JH, et al: Phase II study of carboplatin, irinotecan, and bevacizumab for bevacizumab naïve, recurrent glioblastoma. J Neurooncol 2012;107:155164.

11 Francesconi AB, Dupre S, Matos M, Martin D, Hughes BG, Wyld DK, et al: Carboplatin and etoposide combined with bevacizumab for the treatment of recurrent glioblastoma multiforme. J Clin Neurosci 2010;17:970-974.

12 Nghiemphu PL, Liu W, Lee Y, Than T, Graham C, Lai A, et al: Bevacizumab and chemotherapy for recurrent glioblastoma. Neurology 2009;72:1217-1222.

$\checkmark 13$ Wen PY, Macdonald DR, Reardon DA, Cloughesy TF, Sorensen AG, Galanis E, et al: Updated response assessment criteria for high grade gliomas: response assessment in neuro-oncology working group. J Clin Oncol 2010;28:1963-1972.

14 Zuniga RM, Torcuator R, Jain R, Anderson J, Doyle T, Ellika S, et al: Efficacy, safety, and pattern of response and recurrence in patients with recurrent high-grade gliomas treated with bevacizumab and irinotecan. J Neurooncol 2009;91:329-336.

15 Kreisl TN, Kim L, Moore K, Duic P, Royce C, Stroud I, et al: Phase II trial of single-agent bevacizumab followed by bevacizumab plus irinotecan at tumor progression in recurrent gliobastoma. J Clin Oncol 2009;27:740-745.

-16 Friedman HS, Prados MD, Wen PY, Mikkelsen T, Schiff D, Abrey LE, et al: Bevacizumab alone and in combination with irinotecan in recurrent glioblastoma. J Clin Oncol 2009;27:4733-4740.

$>17$ Wong ET, Lok E, Swanson KD, Gautam S, Engelhard HH, Lieberman F, et al: Response assessment of NovoTTF-100A versus best physician's choice chemotherapy in recurrent glioblastoma. Cancer Med 2014, Epub ahead of print.

18 Verhaak RGW, Hoadley KA, Purdom E, Wang V, Qi Y, Wilkerson MD, et al: Integrated genomic analysis identifies clinically relevant subtypes of glioblastoma characterized by abnormalities in PDGFRA, IDH1, EGFR, and NF1. Cancer Cell 2010;17:98-110. 


\section{Case Reports in Neurology}
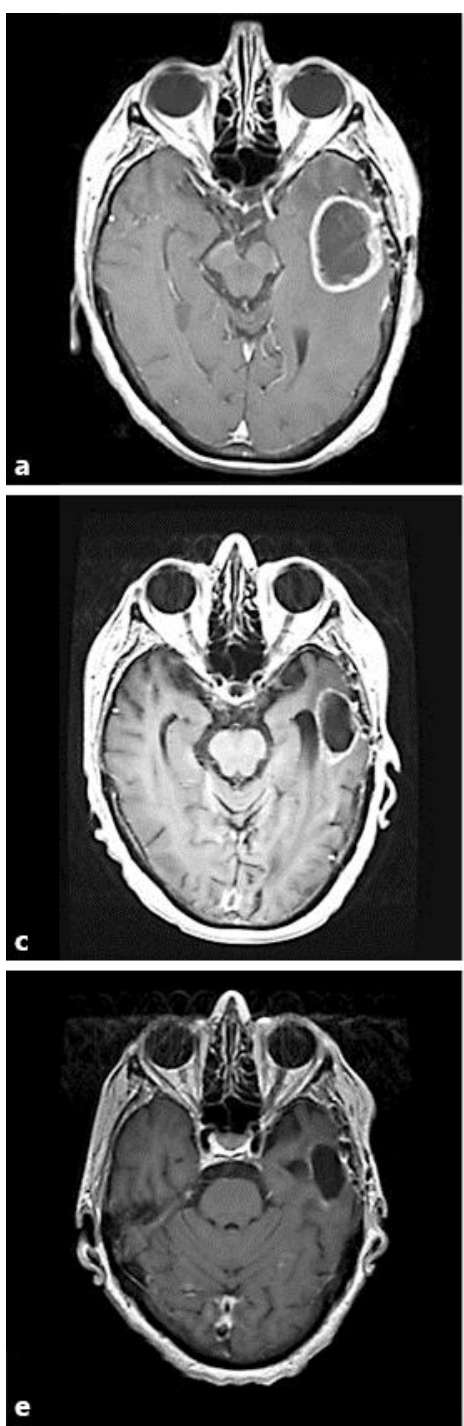

\begin{tabular}{l|l}
\hline Case Rep Neurol 2014;6:109-115 \\
\hline DOI: $10.1159 / 000362264$ & $\begin{array}{l}\text { C 2014 S. Karger AG, Basel } \\
\text { www.karger.com/crn }\end{array}$ \\
\hline
\end{tabular}

Elzinga et al.: Resolution of Cystic Enhancement to Add-On Tumor Treating Electric Fields for Recurrent Glioblastoma after Incomplete Response to Bevacizumab
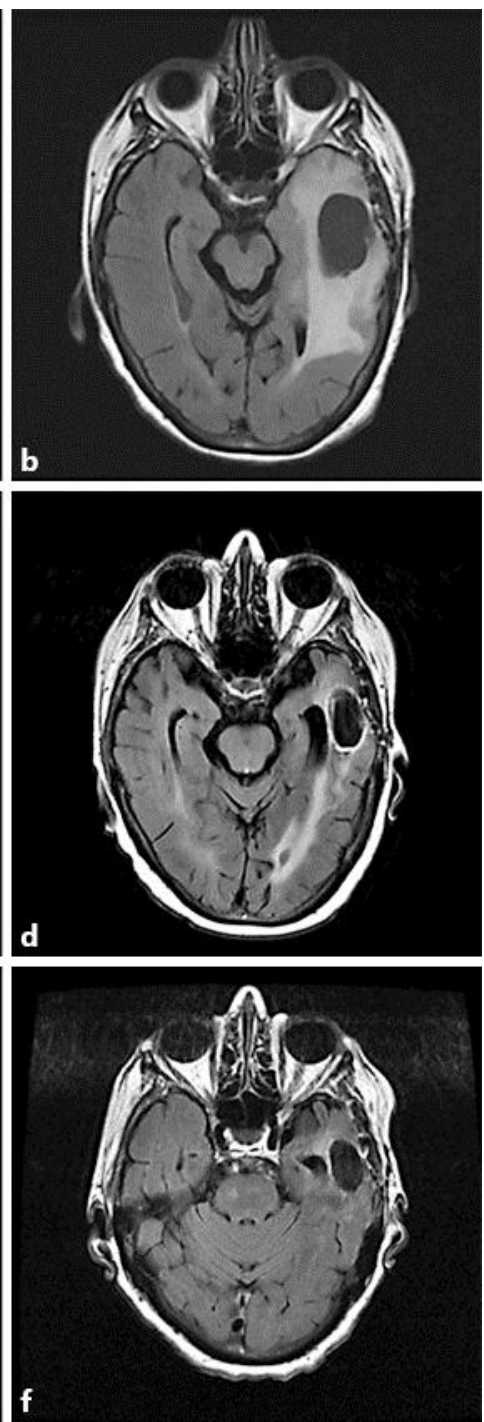

Fig. 1. Response characteristics from combination TTFields and bevacizumab therapy. At the time of first recurrence, the tumor cyst measured $3.4 \times 4.4 \mathrm{~cm}$ on gadolinium-enhanced T1-weighted (a) and FLAIR (b) images. After 2 cycles of bevacizumab, there was a partial decrease in the size of the cystic tumor to $1.9 \times 3.6 \mathrm{~cm}$ as seen on gadolinium-enhanced T1-weighted images (c) and a partial resolution of the edema as detected on FLAIR (d) images. After 6 cycles of combination TTFields and bevacizumab therapy, there was a further decrease of the cystic tumor to $1.7 \times 3.1 \mathrm{~cm}$, or $65 \%$ reduction, as seen on gadoliniumenhanced T1-weighted images (e) and a resolution of edema as detected on FLAIR (f) images. 


\section{Case Reports in Neurology}

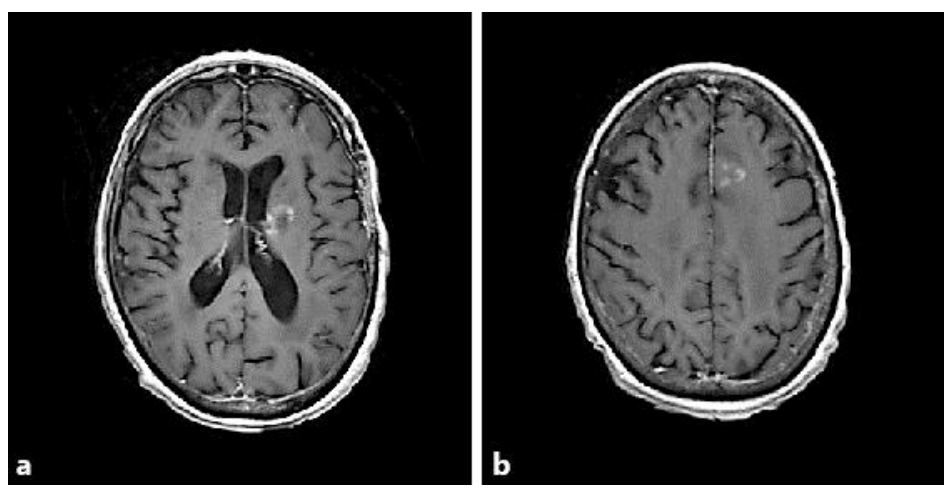

\begin{tabular}{l|l}
\hline DOI: $10.1159 / 000362264$ & $\begin{array}{l}\text { ○ 2014 S. Karger AG, Basel } \\
\text { www.karger.com/crn }\end{array}$ \\
\hline
\end{tabular}

Elzinga et al.: Resolution of Cystic Enhancement to Add-On Tumor Treating Electric Fields for Recurrent Glioblastoma after Incomplete Response to Bevacizumab

Fig. 2. Foci of relapsed disease outside of the previously irradiated glioblastoma. Gadolinium enhancement was detected in the internal capsule (a) and medial left frontal brain (b) after 6 cycles of TTFields and bevacizumab therapy. 